\title{
Quantum Algorithms for Fermionic Simulations
}

\author{
G. Ortiz, J.E. Gubernatis, E. Knill, and R. Laflamme \\ Los Alamos National Laboratory, Los Alamos, NM 87545
}

(February 1, 2008)

\begin{abstract}
We investigate the simulation of fermionic systems on a quantum computer. We show in detail how quantum computers avoid the dynamical sign problem present in classical simulations of these systems, therefore reducing a problem believed to be of exponential complexity into one of polynomial complexity. The key to our demonstration is the spin-particle connection (or generalized Jordan-Wigner transformation) that allows exact algebraic invertible mappings of operators with different statistical properties. We give an explicit implementation of a simple problem using a quantum computer based on standard qubits.

Pacs Numbers: 3.67.Lx, 5.30.-d,
\end{abstract}

Typeset using REVTEX 


\section{INTRODUCTION}

Because of recent exciting algorithms, like the factoring algorithm of Shor [1] and the search algorithm of Grover [2], that solve difficult problems on a quantum computer using algorithms that would be impractical on a classical computer, it is easy to overlook that the original proposals for quantum computers were for the purpose of solving quantum physics problems [3]. People like Feynman [3] focused on the extent to which such a computer could imitate a specific physical process, suggesting in part that quantum problems were inherently too complex for a classical computer [3].

The obvious difficulty with deterministically solving a quantum many-body problem (of fermions or bosons) on a classical computer is the exponentially large basis set needed (i.e., the dimension of its Hilbert space grows exponentially with the number of degrees of freedom). Exact diagonalization approaches (e.g., the Lanczos method) suffer from this exponential "catastrophe". Viewed the other way around, this basis set scaling is what restricts today's classical computer to simulating only small quantum computers. This point seems indisputable, but should not be taken as proof that quantum systems cannot be simulated on a classical computer. By the same token, the recent claim 代 that quantum computers can simulate all quantum systems efficiently lacks explicit and detailed algorithms for specific problems, and lacks a generic model of quantum computation including the unitary maps (quantum gates) that can be physically implementable. Even if a quantum computer existed, some interesting quantum problems, such as finding the ground state of a general quantum Hamiltonian, do not yet have efficient quantum algorithms. Finding such a quantity for small systems is relatively routine on a classical computer.

Feynman in fact analyzed two alternatives for simulating physics with computers $[3]$. One uses a probabilistic classical computer that would produce from the same input as given to a physical system the same distribution of outputs as observed for the physical system. The

other uses a computer constructed of distinctively quantum mechanical elements that obey the laws of quantum mechanics. This latter proposal is the quantum computer. 
To the question, "Can quantum systems be probabilistically simulated by a classical computer?", Feynman's answer was unequivocally "No." 円 This answer is surprising for even at that time some quantum systems were being very successfully simulated probabilistically on classical computers, mainly by quantum Monte Carlo (QMC) methods [5]. To the question, "Can quantum systems be simulated with a quantum computer?", his answer was a qualified "Yes." He believed almost certainly that this could be done for a system of bosons but was unsure that it could be done for a system of fermions. In this paper we present a design for a universal quantum computer that will simulate a system of fermions. Before doing so, we first discuss some problems that can be solved by a probabilistic simulation of a quantum system on a classical computer and others that cannot.

Probabilistic simulations of quantum systems on a classical computer are mainly performed with the use of the Monte Carlo method. These statistical approaches were introduced to overcome the difficulty of exponentially growing phase spaces by numerically evaluating the accompanying many-dimensional integrals by sampling from a function assumed to be non-negative. On a classical computer one can probabilistically simulate a quantum system like liquid $\mathrm{He}^{4}$ [6] and produce results that accurately compare with experiment. The situation, however, is far from satisfactory. An unsatisfactory state of affairs results from the frequent breakdown of the non-negativity assumption and is called "the sign problem." The sign problem is manifested by the seemingly exponentially hard task of estimating the expectation value of an observable with a given error. Interestingly, Feynman's negativism about quantum systems being probabilistically simulated by classical computers was a claim that negative probabilities were unavoidable because of the "hidden variable" problem and the possible violation of Bell inequalities. The extent to which the sign problem is a hidden variable problem is unclear. On the other hand, QMC methods do not faithfully adhere to Feynman's idea of a probabilistic computer. Two important differences

\footnotetext{
${ }^{1}$ There is as yet no mathematical proof that this is the correct answer.
} 
are that most QMC simulations are non-local and performed in imaginary time. Feynman discussed real-time simulations on a local computer. Implications of these differences have been noted by Ceperley [7] who suggests Feynman really argues just against simulating quantum dynamics on a local classical computer. In any case, probabilistic simulations on a classical computer clearly do not qualify as a universal computational scheme for general quantum many-body problems. The limiting factors, for whatever reasons, are negative or complex-valued probabilities whether the simulations are done in real or imaginary time.

To place the sign problem in a better perspective, we will start with a real-time analysis of a collection of interacting quantum particles. Quantum mechanics tells us that these particles either obey Bosonic statistics, whereby the wave function is symmetric with respect to the exchange of the states of any two particles, or obey Fermionic statistics, whereby the wave function is antisymmetric (changes sign) with respect to the exchange of any two particles [8]. Examples of bosons are photons and gluons; examples of fermions are electrons, protons, neutrons, and quarks. Often these two quantum statistics conveniently and efficiently map onto a third, quantum spin statistics [9]. Still in other cases, when particle exchange is unlikely, particle statistics is simply ignored.

For a given initial quantum state $|\Psi(0)\rangle$, a quantum computer solves the time-dependent Schrödinger equation

$$
i \hbar \frac{\partial|\Psi\rangle}{\partial t}=H|\Psi\rangle
$$

by incrementally propagating the initial state via

$$
|\Psi(t)\rangle=\underbrace{e^{-i \Delta t H / \hbar} e^{-i \Delta t H / \hbar} \cdots e^{-i \Delta t H / \hbar}}_{M \text { factors }}|\Psi(0)\rangle .
$$

( $t=M \Delta t$ and the Hamiltonian $H$ is assumed time independent). It should be reasonably apparent that if the Monte Carlo method is applied to the evaluation of the right-hand side of this equation, it is faced with sampling from oscillatory integrands that are not always positive and have unknown nodal surfaces. Further, as time $t$ increases, the integrand fluctuates with increasing rapidity. While clever stationary-phase forms of the QMC method 
have been developed, acceptable solutions are possible only for relatively short times. This form of the sign problem is called the dynamical sign problem, and we are unaware of any efficient [10] real-time QMC simulations for bosonic, fermionic, or quantum spin systems.

Years ago [5], before quantum computers were proposed, it was realized that by transforming Schrödinger's equation to imaginary-time $\tau$ via $t \rightarrow-i \hbar \tau$ the problem with the rapid fluctuations was eliminated. With this transformation, called Wick's rotation, one solves the diffusion-like equation

$$
\frac{\partial|\Psi\rangle}{\partial \tau}=-H|\Psi\rangle
$$

by incrementally propagating the initial state via

$$
|\Psi(\tau)\rangle=\underbrace{e^{-\Delta \tau H} e^{-\Delta \tau H} \cdots e^{-\Delta \tau H}}_{M \text { factors }}|\Psi(0)\rangle .
$$

( $\tau=M \Delta \tau$ and the Hamiltonian $H$ is assumed time independent.) This transformation permits QMC simulations of time-reversal invariant interacting boson systems to a high degree of accuracy. For systems of interacting quantum spins and fermions (or bosons with complex hermitian Hamiltonians [1]), the transformation does not solve the sign problem. For quantum spin systems, the difficulty is finding a basis in which all matrix elements of the positive-definite operator $\exp (-\Delta \tau H)$ are positive. Most notably this difficulty occurs for frustrated quantum spins. For fermion systems, the problem is the Monte Carlo process causing state exchanges that because of the anti-symmetrization requirement just happen to produce samples which are as frequently positive as negative. For the sign problem found in both types of systems, the statistical error of the measured observables grows exponentially fast with increasing system size. Another difficulty with the imaginary-time approach is analytically continuing the results back to real-time if real-time, i.e., truly dynamical, information is needed [12]. This continuation is an ill-posed problem whose solution places extraordinary demands on the simulation [13].

In this paper, we will focus on the dynamical sign problem for a system of fermions, seemingly the most challenging case. Eventually we will give a detailed implementation of a 
simulation of the dynamical properties of a collection of interacting fermions on a quantum computer. The implementation avoids the sign problem. First, in Section III we will discuss more fully the mathematical origin of the dynamical sign problem in classical computation and show why a quantum algorithm overcomes the problem. In Section III we will give the elements required for Deutsch's quantum network model of a quantum computer [14]. The quantum gate in this model conveniently allows the propagation of systems of local two state objects, e.g., a localized quantum spin- $\frac{1}{2}$ particle called qubit. We also propose a universal set of quantum gates (unitary operators) that allows generic propagation of systems of fermions (the fabled "Grassmann chip" [15]). The resulting fermion algebra has been the main technical tool for studying the classical Ising model in two spatial dimensions [16], a prototype lattice model that had an enormous impact on our understanding of phase transitions. Next, In Section IV, we show how this propagation can be effected by the quantum spin gate. We will demonstrate the polynomial scaling of the construction of the initial state, its subsequent time propagation, and the measurement of some observable. Here we will also demonstrate the control of the error in the results. In Section $\square$, we apply our model of dynamical fermion computation to a toy problem to illustrate our procedures in more detail. The exact solution of this toy problem will be given in the Appendix; however, if one were to solve this problem in the obvious Hilbert space, its solution would require a quantum computer for a sufficiently large system. Finally, in Section VI, we summarize and make some remarks about future research directions.

Our universal fermion gate and its mapping to the standard universal quantum gate is very similar to the one recently discussed by Bravyi and Kitaev [17] who actually propose that a quantum computer built from fermions might be more efficient than one built from distinguishable two state systems. 


\section{DYNAMICAL SIGN PROBLEM}

In order to understand the mathematical origin of the dynamical sign problem we use the Feynman path integral formulation [18] for continuum systems in the first quantization representation. In this formalism one maps a quantum problem in $D$ dimensions into a classical one in $D+1$ dimensions and then simulates that problem probabilistically on a classical computer. The algorithm is efficient except for the repetition needed to obtain sufficiently good statistics. The "distinguishable particle" quantum mechanical propagator of a system represented by the Hamiltonian $H=\frac{1}{2} \sum_{i=1}^{N_{e}} p_{i}^{2}+V(\mathcal{R})$ is expressed as 19

$$
G\left(\mathcal{R} \rightarrow \mathcal{R}^{\prime} ; t\right)=\left\langle\mathcal{R}^{\prime}, t\left|e^{-i H t}\right| \mathcal{R}, 0\right\rangle=\int_{\mathcal{R}(0)=\mathcal{R}}^{\mathcal{R}(t)=\mathcal{R}^{\prime}} \mathcal{D}[\mathcal{R}(t)] e^{i S[\mathcal{R}(t)]}
$$

where the measure $\mathcal{D}[\mathcal{R}(t)]=\lim _{M \rightarrow \infty}(2 \pi i t / M)^{-M D / 2} d \mathcal{R}_{1} \cdots d \mathcal{R}_{M-1}$, and the action

$$
S[\mathcal{R}(t)]=\int_{0}^{t} d \tau\left\{\frac{1}{2}\left(\frac{d \mathcal{R}(\tau)}{d \tau}\right)^{2}-V(\mathcal{R}(\tau))\right\} .
$$

Bosonic or fermionic statistics are introduced by applying the corresponding symmetrization operator to the propagator, Eq. 5. However, because the dynamical sign problem occurs for any particle statistics, we will ignore particle statistics for the sake of simplicity.

The description of the properties of different physical systems in terms of correlations of physical observables is the natural way to compare with available experimental information. In this regard, linear response theory provides a way to compute the response of a system to a weak external dynamical perturbation 20]. This linear response is always expressed in terms of a time correlation function of the dynamical variables that couple to the perturbation. For example, if we were to apply an external time-dependent magnetic field and we wanted to calculate the average induced magnetization, we would have to compute a time-dependent magnetization-magnetization correlation function. The two-time correlation function between arbitrary local dynamical variables $A$ and $B$ is

$$
C_{A B}(t)=\langle A(t) B(0)\rangle=\left\langle e^{i H t} A e^{-i H t} B\right\rangle,
$$

if the Hamiltonian is time independent. Expressed in integral form 


$$
C_{A B}(t)=\frac{\int d \mathcal{R} d \mathcal{R}^{\prime} d \mathcal{R}^{\prime \prime} \rho\left(\mathcal{R}, \mathcal{R}^{\prime \prime}\right) G\left(\mathcal{R}^{\prime} \rightarrow \mathcal{R}^{\prime \prime} ;-t\right) A\left(\mathcal{R}^{\prime}\right) G\left(\mathcal{R} \rightarrow \mathcal{R}^{\prime} ; t\right) B(\mathcal{R})}{\int d \mathcal{R} d \mathcal{R}^{\prime} d \mathcal{R}^{\prime \prime} \rho\left(\mathcal{R}, \mathcal{R}^{\prime \prime}\right) G\left(\mathcal{R}^{\prime} \rightarrow \mathcal{R}^{\prime \prime} ;-t\right) G\left(\mathcal{R} \rightarrow \mathcal{R}^{\prime} ; t\right)}
$$

where the "distinguishable particle" density matrix $\rho$ specifies the way the system was initially prepared. The next step consists of writing Eq. 8 in path-integral form using Eq. 5

$$
C_{A B}(t)=\frac{\int d \mathcal{R}_{1} \cdots d \mathcal{R}_{2 M} A\left(\mathcal{R}_{M+1}\right) B\left(\mathcal{R}_{1}\right) P\left(\mathcal{R}_{1}, \cdots, \mathcal{R}_{2 M}\right) e^{i \Phi\left(\mathcal{R}_{1}, \cdots, \mathcal{R}_{2 M}\right)}}{\int d \mathcal{R}_{1} \cdots d \mathcal{R}_{2 M} P\left(\mathcal{R}_{1}, \cdots, \mathcal{R}_{2 M}\right) e^{i \Phi\left(\mathcal{R}_{1}, \cdots, \mathcal{R}_{2 M}\right)}},
$$

where $P$ and $\Phi$ are real-valued functions. Generically, a stochastic estimate of $C_{A B}(t)$ is

$$
C_{A B}(t)=\frac{\sum_{\left\{\mathcal{R}_{i}\right\}} A\left(\mathcal{R}_{M+1}\right) B\left(\mathcal{R}_{1}\right) e^{i \Phi\left(\left\{\mathcal{R}_{i}\right\}\right)}}{\sum_{\left\{\mathcal{R}_{i}\right\}} e^{i \Phi\left(\left\{\mathcal{R}_{i}\right\}\right)}}=\frac{\left\langle A\left(\mathcal{R}_{M+1}\right) B\left(\mathcal{R}_{1}\right) e^{i \Phi\left(\left\{\mathcal{R}_{i}\right\}\right)}\right\rangle_{P}}{\left\langle e^{i \Phi\left(\left\{\mathcal{R}_{i}\right\}\right)}\right\rangle_{P}},
$$

where the configurations $\left\{\mathcal{R}_{i}\right\}$ are sampled from the probability distribution $P$ (positive semidefinite measure). One immediately sees that the origin of the dynamical sign problem is the oscillatory phase factor $e^{i \Phi}$ that leads to large phase fluctuations at long times. Manifestly, $\left|\left\langle e^{i \Phi\left(\left\{\mathcal{R}_{i}\right\}\right)}\right\rangle_{P}\right| \rightarrow 0$ in an exponential fashion as $t$ gets larger. Therefore, the total statistical error for the evaluation of $C_{A B}(t)$ grows exponentially with time because of large cancellations both in the numerator and denominator. The so-called "fermion sign problem" is a particular case of this problem when $e^{i \Phi}= \pm 1$ and time is imaginary [21].

Will a quantum computer solve this problem? At first glance it appears that it will. A quantum computer is a physical system, whether a system of fermions or not, and physical systems have no dynamical or fermion sign problems. Furthermore it has been argued that there are means for mapping most physical systems to a quantum computer in such a way that the quantum computer's controlled evolution mimics that of the physical system [3, 22]. A closer look, however, makes the situation less clear. A quantum computer is a computer, and as such it suffers from limited accuracy. More importantly this type of computer predicts results stochastically, meaning each measurement is one member of the ensemble of measurements possible from a distribution specified by the modulus squared of the wave function for the Hamiltonian $H$ modeled by the quantum computer. For a fixed physical time $t>0$, how accurate is an individual measurement, how accurate is the expectation value of these measurements, and how controlled is their estimated variance? Is the level of accuracy and control achievable polynomially with complexity and $t$ ? 
There is an area where a problem similar to the sign problem has been recognized and resolved by quantum computation. Recently it was shown that quantum computation is polynomially equivalent to classical probabilistic computation with an oracle for estimating the value of simple sums of rational numbers called quadratically signed weight enumerators (QWGTs) [23]. In other words, if these sums could be evaluated, one could use them to generate the quantum statistics needed to simulate the desired quantum system. More specifically, what was demonstrated was the obtainability of expectation value of operators in quantum computation by evaluating sums of the form

$$
S(A, B, x, y)=\sum_{b: A b=0}(-1)^{b^{T} B b} x^{|b|} y^{n-|b|},
$$

where $A$ and $B$ are 0-1-matrices with $B$ of dimension $n \times n$ and $A$ of dimension $m \times n$. The variable $b$ in the summand ranges over 0-1-column vectors of dimension $n, b^{T}$ denotes the transpose of $b,|b|$ is the weight of $b$ (the number of ones in the vector $b$ ), and all calculations involving $A, B$ and $b$ are done modulo 2 . The absolute value of $S(A, B, x, y)$ is bounded by $(|x|+|y|)^{n}$. Quantum computation corresponds to the problem of determining the sign of $S(A, \operatorname{lt}(A), k, l)$ with the restrictions of having $\operatorname{dg}(A)=I, k$ and $l$ being positive integers, and $|S(A, \operatorname{lt}(A), k, l)| \geq\left(k^{2}+l^{2}\right)^{n / 2} / 2 \cdot \operatorname{dg}(A)$ is a diagonal matrix formed from the diagonal elements of $A$ and $\operatorname{lt}(A)$ is a lower triagonal matrix formed from the lower triangular elements of $A$. Details of this quantum algorithm can be found in [23].

The main point is that these sums are similar to the numerator of Eq. 10, and attempts to estimate them by random sampling result in exponentially bad signal to noise ratios. In the case of QWGTs, quantum computers can estimate the sum exponentially better than classical computers, but the estimate is not exact. The situation for the dynamical sign problem is similar: Quantum computers cannot obtain exact values for the desired correlation functions, but can obtain estimates sufficiently exact to avoid the sign problem suffered by the known classical algorithms and to yield usable information about the physical models simulated.

In this paper we will show explicitly how the sign problem is avoided in the case of 
simulating fermions. Below we will give a means for translating local fermion Hamiltonians into the Hamiltonians available in the standard model of quantum computation. In contrast to quantum simulations on a classical computer this translation prevents uncontrolled exchange processes that are the dominant source of the fermion sign problem. With respect to the dynamical sign problem, we then argue by using standard error correction analysis developed for the standard model of quantum computing that these gates will enable sufficiently accurate measurements of correlation functions so the accuracy of the average of these measurements will be dominated by the statistical error. The statistical error is problem dependent but polynomially bounded, so that the difficulty associated with phase-weighted averages is eliminated.

\section{MODELS OF QUANTUM COMPUTATION}

The quantum control model of quantum computation assumes the existence of physical systems that can be controlled by modulating the parameters of the system's Hamiltonian $H_{P}$. The control possibilities are abstracted and used to implement specific quantum gates that represent the unitary evolution of the physical system over a time step obtained by specific modulations of the Hamiltonian. In most treatments, the physical systems, together with the gates, are then taken as the abstract model of quantum computation. The quantum control and quantum gate viewpoints are effectively equivalent, but to tie the computational model to the physics simulation problem more closely, we choose to describe quantum computation from the point of view of quantum control; that is, we will assume an $H_{P}$. In this context we begin by giving the standard model of quantum computation and then defining an alternative model based on fermions.

Defining a model of quantum computation consists of giving an algebra of operators, a set of controllable Hamiltonians (Hermitian operators in the algebra), a set of measurable observables, and an initial state of the physical system. In the simplest case, the observables are measured by the method of projective measurements, and the initial state of the physical 
system is an expectation value of the algebra's operators.

\section{A. Standard Model of Quantum Computation}

The standard model of quantum computation (Deutsch's quantum network representation) is based on an assembly of two state systems called qubits, controlled by one- and two-qubit Hamiltonians, and on a measurement process determined by one-qubit observables.

Operator algebra: It is convenient to define the standard model through the algebra of operators acting on the qubits. This algebra is generated by the unit and Pauli matrices $\sigma_{x}$, $\sigma_{y}$ and $\sigma_{z}$ for each qubit $j$,

$$
\mathbb{1}=\left(\begin{array}{ll}
1 & 0 \\
0 & 1
\end{array}\right) ; \sigma_{x}=\left(\begin{array}{cc}
0 & 1 \\
1 & 0
\end{array}\right) ; \sigma_{y}=\left(\begin{array}{cc}
0 & -i \\
i & 0
\end{array}\right) ; \sigma_{z}=\left(\begin{array}{cc}
1 & 0 \\
0 & -1
\end{array}\right) .
$$

These matrices represent quantum operators with mixed commutation relations and span the space of complex-valued $2 \times 2$ matrices. For qubits $j \neq k$, the $\sigma$ 's commute, and for qubits $j=k$, they satisfy the relation $\sigma_{\mu} \sigma_{\nu}+\sigma_{\nu} \sigma_{\mu}=2 \delta_{\mu \nu} 1,(\mu, \nu=x, y, z)$. For a quantum register with $n$ qubits, one may take the operator $\sigma_{\mu}^{j}$ defined in terms of a Kronecker product

$$
\sigma_{\mu}^{j}=\mathbb{1} \otimes \mathbb{1} \otimes \cdots \otimes \underbrace{\sigma_{\mu}}_{j^{t h} \text { factor }} \otimes \cdots \otimes \mathbb{1}
$$

of matrices acting on $n$ two-dimensional linear spaces. Thus $\sigma_{\mu}^{j}$ admits a matrix representation of dimension $2^{n} \times 2^{n}$.

Control Hamiltonians: Control of qubits is achieved by applying Hamiltonians that act on either one or two qubits. A theorem [24,25] in quantum information processing says that a generic operation on a single qubit and any interaction between two qubits is sufficient for building any unitary operation. We take

$$
H_{P}(t)=\sum_{j}\left[\alpha_{x_{j}}(t) \sigma_{x}^{j}+\alpha_{y_{j}}(t) \sigma_{y}^{j}\right]+\sum_{i, j} \alpha_{i j}(t) \sigma_{z}^{i} \sigma_{z}^{j}
$$

where the $\alpha_{\mu}(t)$ are controllable. Ideally, no constraints on the control functions are assumed. However, it is often simpler to design the required control by assuming that only one of the 
$\alpha_{\mu}(t)$ is non-zero at any time. A quantum algorithm for this model of quantum computation consists of prescribing the control functions [26]. A convenient measure of the complexity

of such an algorithm is the integral $\int_{0}^{t} d t^{\prime} \sqrt{\sum_{\mu} \alpha_{\mu}^{2}\left(t^{\prime}\right)}$ (the action of the algorithm). The quantum gates are simply specific unitary evolutions that may be implemented in terms of $H_{P}$. A convenient universal set of gates is given by operators of the form $\exp \left(i \sigma_{\mu}^{i} \pi / 4\right)$ and $\exp \left(i \sigma_{z}^{i} \sigma_{z}^{j} \pi / 8\right)$. In the quantum network representation of the standard model, an algorithm is a specific sequence of these operators applied to the initial state of the qubits.

Initial state: The initial state of the qubits is assumed to be an $n$ term Kronecker product of the state $|0\rangle \equiv\left(\begin{array}{l}1 \\ 0\end{array}\right)$ which is an eigenstate of $\sigma_{z}$ with eigenvalue 1 . The state is completely determined by the expectation values $\left\langle 0\left|\sigma_{\mu_{i}}^{i}\right| 0\right\rangle$, which are 1 if the $\sigma_{\mu_{i}}^{i}$ are all $\sigma_{z}^{i}$ or the identity, and are 0 otherwise. Physically, the initial state has all "spins" up.

Measurement: The final feature of the model of computation is the specific means for extracting information after a sequence of operations has been applied to the initial state. In the standard model, it is always possible to apply a projective (von Neumann) measurement [27] using the observables $\sigma_{z}^{i}$. With this capability, it is unnecessary to give an initial state explicitly, as the desired state can be prepared by using measurement and operations. To learn the expectation of an observable at the end of an algorithm, one repeats the algorithm and measurement procedure many times and averages over the measurements until the desired accuracy is achieved.

For a description of the standard model of quantum computation in terms of quantum Turing machines, see [28]. Quantum networks are discussed in [24]. Introductory descriptions of the standard model may be found in [29,30.

\section{B. Fermion Model of Quantum Computation: Grassmann chip}

Somewhat analogously, we now describe a standard model of fermion computation. For simplicity we only consider spinless fermions, i.e., fermions without internal spin degrees of freedom, although we could have considered more general fermionic algebras with internal 
degrees of freedom [9]. Physically, a system of spinless fermion might be a system of spin- $\frac{1}{2}$ electrons in a magnetic field sufficiently strong to polarize it fully. The basic system of this model is a state (or fermionic mode) that can be occupied by 0 or 1 spinless fermion. We define the model for $n$ such modes.

Operator algebra: We define the model through the algebra of the spinless fermion operators $a_{j}$ and $a_{j}^{\dagger}$ for each qubit $j(j=1, \cdots, n)$, i.e., through the algebra of $2 n$ elements satisfying canonical anticommutation relations

$$
\left\{a_{i}, a_{j}\right\}=0,\left\{a_{i}, a_{j}^{\dagger}\right\}=\delta_{i j}
$$

where $\{A, B\}=A B+B A$ denotes the anticommutator or Jordan product. $a_{j}^{\dagger}\left(a_{j}\right)$ creates (annihilates) a spinless fermion in state (mode) $j$. Each element admits a matrix representation of dimension $2^{n} \times 2^{n}$. The fermion algebra is isomorphic (as a $*$-algebra) to the standard model (or Pauli) algebra. The isomorphism is established through the Jordan-Wigner mapping [31].

Control Hamiltonians: We take

$$
H_{P}=\sum_{j}\left[\alpha_{j}(t) a_{j}+\tilde{\alpha}_{j}(t) a_{j}^{\dagger}\right]+\sum_{i j} \alpha_{i j}(t)\left(a_{i}^{\dagger} a_{j}+a_{j}^{\dagger} a_{i}\right) .
$$

This is a universal Hamiltonian, i.e., any other Hamiltonian for a system of interacting spinless fermions can be generated by it. Physical operators must be (Hermitian) products of bilinear combinations of the creation and annihilation operators, i.e., products of sums of $a_{j}^{\dagger} a_{i}$ for arbitrary $i$ and $j$ modes.

Initial state: The initial state is assumed to be an $n$ term Kronecker product of the state $|0\rangle$ which is an eigenstate of the number operator $a_{j}^{\dagger} a_{j}$ with eigenvalue 0 . The state is completely determined by the expectation values $\left\langle 0\left|a_{j}^{\dagger} a_{j}\right| 0\right\rangle=0$ for all $j$. Physically, the initial state has all modes unoccupied.

Measurement: Measurements can again be performed by using von Neumann's scheme of projective measurements. In Section $\mathbb{\nabla q}$, we will discuss another scheme more appropriate for the physical systems at hand. 
In the next subsection we show how to simulate the fermion model by using the standard

spin- $\frac{1}{2}$ model. In particular it is possible to efficiently map the fermion Hamiltonians to Pauli operators which can be simulated using the control Hamiltonians of the standard model. This establishes that these two models of computation are polynomially equivalent. Here the point of view is similar to the one used for classical models of computation: the simulation of one model by another establishes their equivalence.

\section{FERMION COMPUTATION VIA THE STANDARD MODEL}

In the previous Section we gave the elements required for Deutsch's quantum network model of a quantum computer [14] and proposed a universal set of quantum gates (unitary operators) that allows generic propagation of systems of fermions (the fabled "Grassmann chip" [15]). Here we show how this propagation can be effected by the quantum spin gate. We will demonstrate the polynomial scaling of the construction of the initial state, its subsequent time propagation, and the measurement of some observable. We will also demonstrate the control of the error in the results.

The first step is the observation that the set of $2 n$ matrices $\gamma_{\mu}$ (of dimension $2^{n} \times 2^{n}$ ) satisfying the Clifford algebra identities

$$
\left\{\gamma_{\mu}, \gamma_{\nu}\right\}=2 \delta_{\mu \nu}
$$

admits a representation in terms of Pauli matrices (Brauer-Weyl construction)

$$
\begin{gathered}
\gamma_{1}=\sigma_{x}^{1}, \gamma_{2}=\sigma_{y}^{1} \\
\gamma_{3}=\sigma_{z}^{1} \sigma_{x}^{2}, \gamma_{4}=\sigma_{z}^{1} \sigma_{y}^{2} \\
\gamma_{5}=\sigma_{z}^{1} \sigma_{z}^{2} \sigma_{x}^{3}, \gamma_{6}=\sigma_{z}^{1} \sigma_{z}^{2} \sigma_{y}^{3} \\
\vdots \\
\gamma_{2 n-1}=\left[\prod_{j=1}^{n-1} \sigma_{z}^{j}\right] \sigma_{x}^{n}, \gamma_{2 n}=\left[\prod_{j=1}^{n-1} \sigma_{z}^{j}\right] \sigma_{y}^{n} .
\end{gathered}
$$

The following mapping of fermion operators 


$$
\begin{aligned}
& a_{j} \rightarrow\left(\prod_{i=1}^{j-1}-\sigma_{z}^{i}\right) \sigma_{-}^{j}=(-1)^{j-1} \sigma_{z}^{1} \sigma_{z}^{2} \cdots \sigma_{z}^{j-1} \sigma_{-}^{j}=(-1)^{j-1} \frac{\gamma_{2 j-1}-i \gamma_{2 j}}{2} \\
& a_{j}^{\dagger} \rightarrow\left(\prod_{i=1}^{j-1}-\sigma_{z}^{i}\right) \sigma_{+}^{j}=(-1)^{j-1} \sigma_{z}^{1} \sigma_{z}^{2} \cdots \sigma_{z}^{j-1} \sigma_{+}^{j}=(-1)^{j-1} \frac{\gamma_{2 j-1}+i \gamma_{2 j}}{2}
\end{aligned}
$$

where $\sigma_{ \pm}^{j}=\frac{\sigma_{x}^{j} \pm i \sigma_{y}^{j}}{2}$, defines a $*$-algebra isomorphism to the algebra of operators of the standard model. It is the so-called spin- $\frac{1}{2}$ Jordan-Wigner transformation [31], and has the property that $\hat{n}_{j}=a_{j}^{\dagger} a_{j}=\sigma_{+}^{j} \sigma_{-}^{j}$. We note that $\hat{n}_{j}$ is a "local" particle number (or density) operator and many types of interaction in physical systems are of the form "density times density" which simplifies the simulation as we will see.

It is important to emphasize that the success of our approach depends upon the mapping of algebras (and not of Hilbert spaces). In this regard it is relevant to mention that the transformation just presented is a particular case of a more general set of mappings that we would like to name generalized Jordan-Wigner transformations [9]. It is possible to imagine a quantum computer implemented, for example, with a three state unit $(S=1)$ instead of a qubit. In such a case, these generalized transformations still allow one to simulate fermions or particles with arbitrary statistics.

Two additional comments are in order: The mapping for $a_{j}$ and $a_{j}^{\dagger}$ described above corresponds to a one-dimensional array of spins. The extension to higher spatial dimensions can be done [32, 33,9] in various ways. A straightforward extension to two dimensions is to re-map the sites of a two dimensional array onto a one dimensional string and proceed as before. Also there is nothing special about using the fermion instead of a quantum spin as an alternative model of computation. One could have just as well used the hard core boson [9]. The main question is whether different algebras admit a physical realization. For hard-core bosons this realization is $\mathrm{He}^{4}$ atoms. 


\section{A. Evolution}

Given a fermion model algorithm, it is necessary to efficiently obtain a corresponding standard model algorithm that at least approximates the desired evolution. The general principle is to map the time-dependent fermion Hamiltonian $H(t)=\sum_{i} H_{i}$ to the standard model operators via the Jordan-Wigner transformation, express the result in terms of a sum of simple products of Pauli operators, and then use the Trotter approximation

$$
e^{-i \Delta t\left(H_{0}+H_{1}+\ldots\right) / \hbar}=\prod_{i} e^{-i \Delta t H_{i} / \hbar}+\mathcal{O}\left((\Delta t)^{2}\right) .
$$

Each time step $\Delta t$ is chosen so that the final error of the simulation is sufficiently small. Provided that the number of terms in the sum is polynomially bounded in the number $n$ of qubits or fermionic modes and provided that each term can be polynomially simulated, the simulation is efficient in $n$ and 1 /error.

To see how to do the simulation, consider the example of the bilinear operator $H_{c}=$ $a_{1} a_{j}^{\dagger}+a_{j} a_{1}^{\dagger}$ in the control Hamiltonian of the fermion model:

$$
\begin{aligned}
H_{c} & =(-1)^{j}\left[\sigma_{-}^{1} \sigma_{z}^{1} \cdots \sigma_{z}^{j-1} \sigma_{+}^{j}+\sigma_{z}^{1} \sigma_{+}^{1} \sigma_{z}^{2} \cdots \sigma_{z}^{j-1} \sigma_{-}^{j}\right] \\
& =\frac{(-1)^{j}}{2}\left[\sigma_{x}^{1} \sigma_{z}^{2} \cdots \sigma_{z}^{j-1} \sigma_{x}^{j}+\sigma_{y}^{1} \sigma_{z}^{2} \cdots \sigma_{z}^{j-1} \sigma_{y}^{j}\right] .
\end{aligned}
$$

It is readily checked that the Jordan-Wigner transformation for the other terms in the control Hamiltonians are also decomposable into sums of a few products of Pauli operators.

The whole idea of a quantum computer is simulating the operations we want by using unitary matrices $U=\exp \left(-i \Delta t H_{P} / \hbar\right)$. These unitary matrices, representing quantum gates, perform reversible computation and are case-dependent. For our particular case, we know

how to simulate $H=\sigma_{z}^{1}$ in the spin- $\frac{1}{2}$ case (it is directly implemented in the standard model), so we ask what set of unitary operations produce the evolution $\tilde{U}=\exp \left(-i \Delta t H_{c} / \hbar\right)$. In other words, how do we write a $U=U_{1} \ldots U_{k}$ such that $H_{c}=U^{\dagger} H U$ ? Consider for example the Hamiltonian $H_{x}=\sigma_{x}^{1} \sigma_{z}^{2} \cdots \sigma_{z}^{j-1} \sigma_{x}^{j}$. The procedure is as follows: The unitary operator

$$
U_{1}=e^{i \frac{\pi}{4} \sigma_{y}^{1}}=\frac{1}{\sqrt{2}}\left[\hat{\mathbb{1}}+i \sigma_{y}^{1}\right]=\frac{1}{\sqrt{2}}\left(\begin{array}{cc}
1 & 1 \\
-1 & 1
\end{array}\right) \otimes \mathbb{1} \otimes \cdots \otimes \mathbb{1}
$$


takes $\sigma_{z}^{1} \rightarrow \sigma_{x}^{1}$, i.e., $U_{1}^{\dagger} \sigma_{z}^{1} U_{1}=\sigma_{x}^{1}$. The operator

$$
U_{2}=e^{i \frac{\pi}{4} \sigma_{z}^{1} \sigma_{z}^{2}}=\frac{1}{\sqrt{2}}\left[\hat{1}+i \sigma_{z}^{1} \sigma_{z}^{2}\right]
$$

takes $\sigma_{x}^{1} \rightarrow \sigma_{y}^{1} \sigma_{z}^{2}$. The next step is

$$
U_{3}=e^{i \frac{\pi}{4} \sigma_{z}^{1} \sigma_{z}^{3}}
$$

to take $\sigma_{y}^{1} \sigma_{z}^{2} \rightarrow-\sigma_{x}^{1} \sigma_{z}^{2} \sigma_{z}^{3}$. By successively similar steps we easily build the required string of operators: $\sigma_{x}^{1} \sigma_{z}^{2} \cdots \sigma_{z}^{j-1} \sigma_{x}^{j}$.

If $j$ is odd,

$$
U_{j}=e^{i \frac{\pi}{4} \sigma_{z}^{1} \sigma_{z}^{j}}
$$

will take $\sigma_{y}^{1} \sigma_{z}^{2} \cdots \sigma_{z}^{j-1} \rightarrow(-1)^{\left[\frac{j-1}{2}\right]} \sigma_{x}^{1} \sigma_{z}^{2} \cdots \sigma_{z}^{j}$, where $\left[\frac{m}{l}\right]$ is the integer part of $m / l$. The final operator

$$
U_{j+1}=e^{i \frac{\pi}{4} \sigma_{y}^{j}}
$$

will bring the control operator to the desired one (up to a global phase $(-1)^{\left[\frac{j-1}{2}\right]}$ ):

$$
\sigma_{x}^{1} \sigma_{z}^{2} \cdots \sigma_{z}^{j-1} \sigma_{x}^{j}
$$

If $j$ is even, we need an additional unitary operator that flips the first qubit's $\sigma_{y}^{1}$ into a $\sigma_{x}^{1}$. This flip is achieved with the operator

$$
U_{j+2}=e^{-i \frac{\pi}{4} \sigma_{z}^{1}}=\left(\begin{array}{cc}
e^{-i \frac{\pi}{4}} & 0 \\
0 & e^{i \frac{\pi}{4}}
\end{array}\right) \otimes \mathbb{1} \otimes \cdots \otimes \mathbb{1}
$$

that takes $\sigma_{y}^{1} \rightarrow \sigma_{x}^{1}$.

Hence, to construct this non-local fermion operator from the standard model requires additional steps that are proportional to $j$. This number scales polynomially with the complexity so the construction is efficient if the standard model is efficient.

The one and two-body nature of naturally occurring interactions means that a term in a second-quantized representation of a Hamiltonian only has one of two forms: either $a_{i}^{\dagger} a_{j}$ or $a_{i}^{\dagger} a_{j} a_{k}^{\dagger} a_{l}$. We just demonstrated how to handle the first case. The second case merely requires applying that algorithm twice. This squares the complexity. 


\section{B. State preparation}

In this Section we discuss the preparation of states of physical relevance. Clearly, the preparation of the initial state is a very important step since the study and efficiency of the given physical process one wants to simulate depends upon it.

Consider a system of $N_{e}$ fermions and $n$ operators $a_{j}^{\dagger}$ (single particle states). A generic $N_{e}$-particle state of a Hilbert space $\mathcal{H}_{N_{e}}$ of antisymmetrized wave functions can always be expanded in terms of the antisymmetric states

$$
\left|\Phi_{\alpha}\right\rangle=\prod_{j=1}^{N_{e}} b_{j}^{\dagger}|\mathrm{vac}\rangle
$$

where $b_{j}^{\dagger}$ creates a state $j$ and $|\mathrm{vac}\rangle=|0\rangle \otimes|0\rangle \cdots \otimes|0\rangle$ is the vacuum state (i.e., $b_{j}|0\rangle=0$, $\forall j)$. The operator $b^{\dagger}$ is in general a linear combination of $a^{\dagger}$ 's, i.e., $b_{j}^{\dagger}=\sum_{i=1}^{n} a_{i}^{\dagger} P_{i j}$ where $P_{i j}$ is some matrix and $N_{e} \leq n$.

The states $\left|\Phi_{\alpha}\right\rangle\left(\alpha=1, \cdots,\left(\begin{array}{c}n \\ N_{e}\end{array}\right)\right)$ in general form an overcomplete set of nonorthogonal states that span the whole $\mathcal{H}_{N_{e}}$, i.e., redundantly generate $\mathcal{H}_{N_{e}}$. They are known as Slater determinants [20]. Typically, $\left|\Phi_{\alpha}\right\rangle$ is the result of a self-consistent mean-field (or generalized Hartree-Fock) calculation. Even a Bardeen-Cooper-Schrieffer superconducting state, which does not preserve the number of particles, can be written in this way after an appropriate canonical transformation which redefines the vacuum state [34].

One can easily prepare the states $\left|\Phi_{\alpha}\right\rangle$ noticing that the quantum gate, represented by the unitary operator

$$
U_{m}=e^{i \frac{\pi}{2}\left(b_{m}+b_{m}^{\dagger}\right)}
$$

when acting on the vacuum state, produces $b_{m}^{\dagger}|0\rangle$ up to a phase $e^{i \frac{\pi}{2}}$. Therefore, the successive application of similar unitary operators will generate the state $\left|\Phi_{\alpha}\right\rangle$ up to a global phase.

Except for very small systems the total Hilbert space is too large to be fully used (it has an exponential growth with increasing system size). In practice, one works in a subspace of $\mathcal{H}_{N_{e}}$ that closely represents the physical state one is trying to simulate. Generically, as initial state, we will consider a very general expression of a many-fermion state: 


$$
|\Psi(t=0)\rangle=\sum_{\alpha=1}^{N} \mathrm{a}_{\alpha}\left|\Phi_{\alpha}\right\rangle
$$

where the integer $N$ is a finite and small number. The state can be prepared efficiently (in $N$ ) by a number of procedures. We now describe one.

To make the description simple, we will assume $\sum_{\alpha=1}^{N}\left|\mathrm{a}_{\alpha}\right|^{2}=1$ and $\left\langle\Phi_{\alpha} \mid \Phi_{\beta}\right\rangle=\delta_{\alpha \beta}$, which is equilvalent to requiring $\left\{\left|\Phi_{\alpha}\right\rangle\right\}$ to be an orthonormal set and $|\Psi(t=0)\rangle$ to be normalized to unity. With these assumptions the steps of the state preparation algorithm are:

1. Adjoin $N$ auxiliary (ancilla) qubits, each in the state $|0\rangle$, to the vacuum of the physical system. The resulting state is

$$
\underbrace{|0\rangle \otimes|0\rangle \otimes \cdots|0\rangle}_{N} \otimes|\mathrm{vac}\rangle \equiv|0\rangle_{\mathrm{a}} \otimes|\mathrm{vac}\rangle
$$

2. From this state generate $\sum_{\alpha=1}^{N} \mathrm{a}_{\alpha}|\alpha\rangle \otimes \mid$ vac $\rangle$ where $|\alpha\rangle$ is an ancilla state with only the $\alpha$ qubit being $|1\rangle$. The procedure for generating this combination of states is described below.

3. For each $\alpha=1, \ldots, N$, conditional on the $\alpha$ qubit being $|1\rangle$, apply the state preparation procedure for $\left|\Phi_{\alpha}\right\rangle$. The resulting state is

$$
\sum_{\alpha=1}^{N} \mathrm{a}_{\alpha}|\alpha\rangle \otimes\left|\Phi_{\alpha}\right\rangle
$$

4. From this state generate

$$
\frac{1}{\sqrt{N}} \sum_{\alpha=1}^{N} \mathrm{a}_{\alpha}|0\rangle_{\mathrm{a}} \otimes\left|\Phi_{\alpha}\right\rangle+\text { terms without }|0\rangle_{\mathrm{a}}
$$

This step will also be described below.

The final step is accepted if a measurement shows all the ancillas being returned to $|0\rangle_{\mathrm{a}}$. The probability of successful preparation is thus $\sum_{\alpha=1}^{N}\left|\mathrm{a}_{\alpha}\right|^{2} / N=1 / N$. Consequently, on average, $N$ trials will be needed before a successful state preparation. 
The procedure to produce step 2 is most easily described by example. We will assume $N=2$. The problem then is to generate $\mathrm{a}_{1}|10\rangle \otimes|\mathrm{vac}\rangle+\mathrm{a}_{2}|01\rangle \otimes|\operatorname{vac}\rangle$ from $|00\rangle \otimes|\mathrm{vac}\rangle$. In what follows all operations will be only on the ancilla part of the initial state so we will not explicitly show the vacuum. We also note that one can always apply a rotation to a given qubit that will take $|0\rangle$ into $x|0\rangle+y|1\rangle$ with $|x|^{2}+|y|^{2}=1$. The steps of the procedure are:

2.1 Adjoin an ancilla qubit $|\mathrm{b}\rangle$ initially being $|0\rangle$. The initial state is now $|0\rangle \otimes|00\rangle$.

2.2 Conditional on $|\mathrm{b}\rangle=|0\rangle$, rotate the $\alpha=1$ qubit, and then conditional on the $\alpha=1$ qubit being $|1\rangle$, flip $|\mathrm{b}\rangle$ :

$$
x_{1}|0\rangle \otimes|00\rangle+y_{1}|1\rangle \otimes|10\rangle
$$

2.3 Conditional on $|\mathrm{b}\rangle$ being $|0\rangle$, rotate the $\alpha=2$ qubit, and then conditional on the $\alpha=2$ qubit being $|1\rangle$, flip $|\mathrm{b}\rangle$ :

$$
x_{1} x_{2}|0\rangle \otimes|00\rangle+x_{1} y_{2}|1\rangle \otimes|01\rangle+y_{1}|1\rangle \otimes|10\rangle
$$

2.4 Project out the states with $|\mathrm{b}\rangle$ being $|1\rangle$ :

$$
x_{1} y_{2}|01\rangle+y_{1}|10\rangle
$$

The rotations are chosen so that $\mathrm{a}_{1}=y_{1}$ and $\mathrm{a}_{2}=x_{1} y_{2}$.

For the explanation of step 4, we will display the physical states. The problem is: Starting with $\mathrm{a}_{1}|10\rangle \otimes\left|\Phi_{1}\right\rangle+\mathrm{a}_{2}|01\rangle \otimes\left|\Phi_{2}\right\rangle$, produce (16).

4.1 Adjoin an ancilla qubit $|\mathrm{b}\rangle$ initially being $|0\rangle$. The initial state is now $\mathrm{a}_{1}|0\rangle \otimes|10\rangle \otimes$ $\left|\Phi_{1}\right\rangle+\mathrm{a}_{2}|0\rangle \otimes|01\rangle \otimes\left|\Phi_{2}\right\rangle$.

4.2 Conditional on $|\mathrm{b}\rangle$ being $|0\rangle$, rotate the $\alpha=1$ qubit, and then conditional on the $\alpha=1$ qubit being $|1\rangle$, flip $|b\rangle$ :

$$
\mathrm{a}_{1}\left(x_{1}|0\rangle \otimes|00\rangle+y_{1}|1\rangle \otimes|10\rangle\right) \otimes\left|\Phi_{1}\right\rangle+\mathrm{a}_{2}\left(x_{1}|0\rangle \otimes|01\rangle+y_{1}|1\rangle \otimes|11\rangle\right) \otimes\left|\Phi_{2}\right\rangle
$$


4.3 Conditional on $|\mathbf{b}\rangle$ being $|0\rangle$, rotate the $\alpha=2$ qubit, and then conditional on the $\alpha=2$ qubit being $|1\rangle$, flip $|b\rangle$ :

$$
\mathrm{a}_{1} x_{1}\left(x_{2}|0\rangle \otimes|00\rangle+y_{2}|1\rangle \otimes|01\rangle\right) \otimes\left|\Phi_{1}\right\rangle+\mathrm{a}_{2} x_{1}\left(x_{2}|0\rangle \otimes|00\rangle+y_{2}|1\rangle \otimes|01\rangle\right) \otimes\left|\Phi_{2}\right\rangle
$$

4.4 Project out the states with $|\mathrm{b}\rangle=|0\rangle$ :

$$
x_{1} x_{2}\left(\mathrm{a}_{1}|00\rangle \otimes\left|\Phi_{1}\right\rangle+\mathrm{a}_{2}|00\rangle \otimes\left|\Phi_{2}\right\rangle\right)
$$

The rotations are chosen so that $x_{1} x_{2}$ equals $1 / \sqrt{N}$ where $N=2$. Comparing step 2 with step 4, one sees they are structurally identical, differing by the set of amplitudes generated and the complementarity of the subspaces selected for the final result. This latter difference in some sense makes one procedure the inverse of the other. For the case of $N>2$, one simply replaces steps 2.2 and 2.3 and steps 4.2 and 4.3 by "do loops" over $\alpha$ from 1 to $N$.

On average, the entire procedure needs $N$ trials before a successful state preparation. (In many cases, the other measurement outcomes can be used also to avoid too many trials.) Construction of the initial state thus scales as $\mathcal{O}\left(N^{2} n N_{e}\right) \leq \mathcal{O}\left(N^{2} n^{2}\right)$ so unless the number of Slater determinants is exponentially large, general many-fermion states can be initialized efficiently.

\section{Measurement}

While there is a variety of physical observables one measures experimentally and calculates theoretically, at this time it is difficult to demonstrate that they all can be computed efficiently on a quantum computer. Fortunately, we will now argue that one important class of observables, the temporal correlation functions $C_{A B}(t)$, can be computed not only efficiently but also accurately. These functions describe the temporal evolution of some observable $A(t)$ in response to some weak external stimulus that couples to the system's variable $B(0)$. They are at the heart of understanding, for example, the optical properties of materials. 
The goal is to determine correlations of the form $C_{A B}(t)=\langle A(t) B(0)\rangle=\left\langle e^{i H t} A e^{-i H t} B\right\rangle$ up to a sufficiently small statistical error. Clearly, measuring efficiently $C_{A B}$ is not possible for an arbitrary $A$ and $B$. One sufficient condition is that $A$ and $B$ are efficiently simulatable Hamiltonians. This observation is based on a method for determining $C_{A B}$ refined by Kitaev [35] and applied to the measurement of correlation functions by Terhal and DiVincenzo [36]. Here we give a different method based on an idea given in [37].

A general principle that can be used to obtain $C_{A B}$ is to decompose the operator whose expectation needs to be determined, i.e., $A(t) B(0)$, into a small sum of operators of a simpler form and measure each summand individually. Our method directly measures expectations of the form $\left\langle U^{\dagger} V\right\rangle$ when algorithms for implementing the unitary operators $U$ and $V$ are available. General correlation functions are then determined by decomposing operators using a unitary operator basis, for example the one consisting of products of Pauli matrices.

The method for measuring $\left\langle U^{\dagger} V\right\rangle$ consists of the following steps:

1. Adjoin via a direct product an ancilla (i.e., an auxiliary) qubit a in the state $(|0\rangle+$ $|1\rangle) / \sqrt{2}$ with density matrix $\rho_{\mathbf{a}}=\left(\mathbb{1}+\sigma_{x}^{\mathbf{a}}\right) / 2$ to the initial state of the system described by the density matrix $\rho$.

2. Apply the conditional evolutions $\bar{U}_{1}=|0\rangle\langle 0|\otimes U+| 1\rangle\langle 1| \otimes \mathbb{1}$ and $\bar{U}_{2}=|1\rangle\langle 1| \otimes V+$ $|0\rangle\langle 0| \otimes \mathbb{1}\left(\bar{U}=\bar{U}_{1} \bar{U}_{2}\right)$. The methods of [24] may be used to implement these evolutions given algorithms for $U$ and $V$.

3. Measure $2 \sigma_{+}^{\mathbf{a}}=\sigma_{x}^{\mathbf{a}}+i \sigma_{y}^{\mathbf{a}}=2|0\rangle\langle 1|$. This may be done by measuring $\sigma_{x}^{\mathbf{a}}$ and $\sigma_{y}^{\mathbf{a}}$ in sufficiently many independent trials of these steps.

4. Given the initial density matrix $\rho$, the expectation

$$
\begin{aligned}
\left\langle\sigma_{x}^{\mathbf{a}}+i \sigma_{y}^{\mathbf{a}}\right\rangle_{\rho_{\mathbf{a}} \otimes \rho} & =2 \operatorname{Tr}_{n+1}\left[\bar{U}^{\dagger}|0\rangle\langle 1| \bar{U} \rho_{\mathbf{a}} \otimes \rho\right] \\
& =\operatorname{Tr}_{n+1}\left[|0\rangle\langle 1| \otimes U^{\dagger} V \rho_{\mathbf{a}} \otimes \rho\right]=\operatorname{Tr}_{n}\left[U^{\dagger} V \rho\right]=\left\langle U^{\dagger} V\right\rangle_{\rho},
\end{aligned}
$$

as desired. The statistical noise in the measurement of $\left\langle U^{\dagger} V\right\rangle_{\rho}$ is determined by that of two binary random variables and therefore depends only on the value of $\operatorname{Tr}_{n}\left[U^{\dagger} V \rho\right]$, 
which is inside the unit complex circle. As a result it is a simple matter to determine the number of measurement attempts required to achieve sufficient statistical accuracy.

The procedure for measuring $C_{A B}(t)$ can now be summarized as follows: First express $A=A(0)$ and $B=B(0)$ as a sum of unitary operators $A=\sum_{j=1}^{m_{A}} A_{j}$ and $B=\sum_{j^{\prime}=1}^{m_{B}} B_{j^{\prime}}$. A convenient unitary operator basis that works well for the local observables of interest consists of all the products of Pauli operators, as each such product is easily implemented as a quantum algorithm. Then, for each $j$ and $j^{\prime}$ one uses the just described method with $U=e^{i H t} A_{j}^{\dagger} e^{-i H t}$ and $V=B_{j^{\prime}}$ to obtain $\left\langle A_{j}(t) B_{j^{\prime}}(0)\right\rangle . V$ may be implemented by simulating the evolution under $H$, applying $B_{j^{\prime}}$, and then undoing the evolution under $H$.

An alternative approach to the measurement process is von Neumann's projection method. We sketch it here for completeness and comparison. In this approach we also add an auxiliary (ancilla) degree of freedom to the problem. Suppose that this extra qubit corresponds to an harmonic oscillator degree of freedom $|e\rangle$. Then, we consider the composite state

$$
|\Psi\rangle_{S} \otimes|e\rangle_{0}
$$

where $|\Psi\rangle_{S}=\sum_{j} \lambda_{j}\left|\phi_{j}\right\rangle_{S}$ is the state of the system we want to probe and $|e\rangle_{t}$ is the state of the harmonic oscillator in the coordinate $(x$-)representation. The corresponding state in the momentum ( $p$-)representation is denoted $|\hat{e}\rangle_{t}$.

Assume the observable ( $t$-independent Hermitian operator) we want to measure is $\mathcal{A}$. Then, we are interested in determining ${ }_{S}\langle\Psi|\mathcal{A}| \Psi\rangle_{S}$ in an efficient way. Suppose that we know how to implement the unitary operation $U^{S}(t)=e^{-i \mathcal{A} t}$. Following Kitaev we want to implement the following conditional evolution

$$
\mathcal{U}=\sum_{t}|e\rangle_{t}\langle e| U^{S}(t)
$$

From the spectral theorem we can write $\mathcal{A}=\sum_{j} \Lambda_{j}\left|\phi_{j}\right\rangle_{S}\left\langle\phi_{j}\right|$. Then,

$$
\mathcal{U}\left|\phi_{j}\right\rangle_{S} \otimes|\hat{0}\rangle_{0}=\sum_{t} \mathcal{U}\left|\phi_{j}\right\rangle_{S} \otimes|e\rangle_{t}=\sum_{t} e^{-i \Lambda_{j} t}\left|\phi_{j}\right\rangle_{S} \otimes|e\rangle_{t}=\left|\phi_{j}\right\rangle_{S} \otimes\left|\hat{\Lambda}_{j}\right\rangle_{t}
$$


where $|\hat{0}\rangle_{0}$ is a state with $(p=0)$ zero momentum. Basically, the conditional evolution $\mathcal{U}$ is a momentum translation operator for the harmonic oscillator extra state. Finally,

$$
\mathcal{U}|\Psi\rangle_{S} \otimes|\hat{0}\rangle_{0}=\sum_{j} \lambda_{j}\left|\phi_{j}\right\rangle_{S} \otimes\left|\hat{\Lambda}_{j}\right\rangle_{t}
$$

Although the second measurement method is conceptually simpler, it requires approximately implementing the ancillary harmonic oscillator, the conditional evolutions for many different choices of $t$, and a more complex analysis of the measurement statistics. The conditional evolutions can be simplified somewhat, and in special cases (such as as a subroutine of factoring) become very efficient-see [35].

\section{Measurement Noise Control}

The quantum physics simulation algorithm described above is approximate and the output is noisy. In order to properly use it, we need to have explicit estimates of the error $\epsilon$ in the inferred expectations given the noise in the implementation. Furthermore, the effort required to make $\epsilon$ small must scale polynomially with $1 / \epsilon$. There are three sources of error that need to be considered. The first is associated with intrinsic noise in the implementation of the gates due to imperfections and unwanted interactions. The second comes from the discretization of the evolution operator and the use of the Trotter decomposition. The third is due to the statistics in measuring the desired correlation function using the technique given above.

\section{Gate imperfections}

The problem of gate imperfections can be dealt with by using quantum error correction 38,39 and fault tolerant quantum computation 40 44. According to the accuracy threshold theorem, provided the physical gates have sufficiently low error, it is possible to quantum compute arbitrarily accurately. The fault tolerant computation implements unitary operations and measurements on encoded qubits with overheads bounded by $\mathcal{O}\left(\log ^{k}(1 / \epsilon)\right)$ 
for some $k$. This exponentially efficient convergence implies that the effects of physical noise can in principle be ignored.

\section{Discretization error}

A second type of error is the one introduced by the discretization of the evolution operator. This discretization is very similar to the one used in classical simulation of dynamical quantum systems. It is possible to estimate the size of this error by a detailed analysis of the discretization. For example using the Trotter approximation

$$
e^{-i\left(H_{1}+H_{2}\right) \Delta t}=e^{-i\left(H_{1}\right) \Delta t / 2} e^{-i\left(H_{2}\right) \Delta t} e^{-i\left(H_{1}\right) \Delta t / 2}+\mathcal{O}\left((\Delta t)^{3}\right)
$$

The coefficient of $(\Delta t)^{3} \sim-i\left(H_{1}+H_{2}\right)^{3} / 6$ can be bounded by estimating the largest eigenvalue of $H_{1}$ and $H_{2}$.

\section{Measurement statistics}

Our technique for measuring the correlation function $\langle A(t) B(0)\rangle$ requires measuring the expectations of unitary operators $U_{j}^{\dagger} V_{j^{\prime}}$ associated with the implemented evolution. In most cases, the operators $A$ and $B$ are a sum of $\mathcal{O}\left(m_{A, B}\right)$ products of Pauli matrices, so that $\mathcal{O}\left(m_{A}\right) U_{j}^{\dagger}$ 's and $\mathcal{O}\left(m_{B}\right) V_{j}$ 's are needed. This means that the expectation is a sum of $\mathcal{O}\left(m_{A} m_{B}\right)$ random variables $r_{j j^{\prime}}$, where $\left|r_{j j^{\prime}}\right| \leq 1$. To assure that the statistical noise (given by the standard deviation) is less than $\epsilon$ it suffices to measure each $r_{j j^{\prime}} \mathcal{O}\left(m_{A} m_{B} / \epsilon^{2}\right)$ times.

\section{EXAMPLE: RESONANT IMPURITY SCATTERING}

\section{A. Formulation of the Physical Problem}

Our toy problem is a ring of $n$ equally-spaced lattice sites on which spinless fermions hop to nearest neighbor sites or hop onto or from an "impurity" state. The length of the ring is 
$L=n a$, where $a$ is the distance between sites. The system is described by the Hamiltonian (in second quantized form)

$$
H=-\mathcal{T} \sum_{i=1}^{n}\left(c_{i}^{\dagger} c_{i+1}+c_{i+1}^{\dagger} c_{i}\right)+\epsilon b^{\dagger} b+\frac{V}{\sqrt{n}} \sum_{i=1}^{n}\left(c_{i}^{\dagger} b+b^{\dagger} c_{i}\right),
$$

As usual, $b$ 's and c's are fermion (anticommuting) operators. The index $i$ labels the lattice sites $\left(R_{i}=i a\right.$ is the lattice site position $)$ and strict periodic boundary conditions are assumed, i.e.,

$$
c_{i+n}^{\dagger}=c_{i}^{\dagger} .
$$

We now imagine that the system is initially prepared in the zero temperature ground state of the ring in the absence of the impurity. Then, at time $t=0$, a fermion is injected into the impurity state. After the system has evolved for some time $t$, we want to compute the probability amplitude that the evolved state is still in the initial state. The relevant quantity to compute is $(\hbar=1$ and $t \geq 0)$

$$
\begin{aligned}
G(t) & =\left\langle\Psi(0)\left|b(t) b^{\dagger}(0)\right| \Psi(0)\right\rangle, \\
b(t) & =e^{i H t} b(0) e^{-i H t},
\end{aligned}
$$

where the initial state is the Fermi sea of $N_{e} \leq n$ fermions

$$
|\Psi(0)\rangle=|F S\rangle=\prod_{i=0}^{N_{e}-1} c_{k_{i}}^{\dagger}|0\rangle .
$$

$|0\rangle$ is the vacuum of fermions and

$$
c_{k_{i}}^{\dagger}=\frac{1}{\sqrt{n}} \sum_{j=1}^{n} e^{i k_{i} R_{j}} c_{j}^{\dagger} .
$$

The wave number $k_{j}$ is determined from the periodic boundary conditions, $c_{i+n}^{\dagger}=c_{i}^{\dagger}$, which implies

$$
k_{j}=\frac{2 \pi n_{j}}{L}, \text { with } n_{j} \text { an integer } .
$$

There is no unique way to choose the set of $n_{j}$ 's. The common convention is to define the first Brillouin zone as 


$$
-\frac{\pi}{a}<k \leq \frac{\pi}{a}
$$

with $k$ values uniformly distributed in this interval with spacing $\Delta k=2 \pi / L$.

\section{B. Quantum Algorithm}

We want to write a quantum algorithm that allows one to compute $G(t)$ (see the Appendix for the exact closed-form solution). To this end, we start by representing fermion operators in Eq. A5 in terms of Pauli matrices. Because of the form of the hybridization term, Eq. A4, a most convenient representation is the following

$$
\begin{array}{cc}
b=\sigma_{-}^{1} & b^{\dagger}=\sigma_{+}^{1} \\
c_{k_{0}}=-\sigma_{z}^{1} \sigma_{-}^{2} & c_{k_{0}}^{\dagger}=-\sigma_{z}^{1} \sigma_{+}^{2} \\
\vdots & \vdots \\
c_{k_{n-1}}=(-1)^{n} \sigma_{z}^{1} \sigma_{z}^{2} \cdots \sigma_{z}^{n} \sigma_{-}^{n+1} & c_{k_{n-1}}^{\dagger}=(-1)^{n} \sigma_{z}^{1} \sigma_{z}^{2} \cdots \sigma_{z}^{n} \sigma_{+}^{n+1},
\end{array}
$$

from which the following mapping results

$$
\begin{aligned}
b^{\dagger} b & =2\left(1+\sigma_{z}^{1}\right) \\
c_{k_{i}}^{\dagger} c_{k_{i}} & =2\left(1+\sigma_{z}^{i+2}\right) \\
c_{k_{0}}^{\dagger} b+b^{\dagger} c_{k_{0}} & =\frac{1}{2}\left(\sigma_{x}^{1} \sigma_{x}^{2}+\sigma_{y}^{1} \sigma_{y}^{2}\right) .
\end{aligned}
$$

Therefore, the Hamiltonian operator reads

$$
\frac{H}{2}=\left[\epsilon+\sum_{i=0}^{n-1} \mathcal{E}_{k_{i}}\right] \mathbb{1}+\epsilon \sigma_{z}^{1}+\sum_{i=0}^{n-1} \mathcal{E}_{k_{i}} \sigma_{z}^{i+2}+\frac{V}{4}\left(\sigma_{x}^{1} \sigma_{x}^{2}+\sigma_{y}^{1} \sigma_{y}^{2}\right) .
$$

An additional simplification can be introduced when one realizes that the structure of the observable to be measured is such that

$$
b(t)=e^{i H t} b(0) e^{-i H t}=e^{i \bar{H} t} \sigma_{-}^{1} e^{-i \bar{H} t},
$$

where $\bar{H}$ is given by 


$$
\bar{H}=2 \epsilon \sigma_{z}^{1}+2 \mathcal{E}_{k_{0}} \sigma_{z}^{2}+\frac{V}{2}\left(\sigma_{x}^{1} \sigma_{x}^{2}+\sigma_{y}^{1} \sigma_{y}^{2}\right),
$$

and, therefore, the "string" one has to simulate has length equal to two (it involves only qubits 1 and 2)

$$
\mathcal{A}(t)=b(t) b^{\dagger}(0)=e^{i \bar{H} t} \sigma_{-}^{1} e^{-i \bar{H} t} \sigma_{+}^{1} .
$$

If we were to transform $\bar{H}=U H_{P 1} U^{\dagger}$ unitarily with $U=\prod_{j=1}^{n} e^{i H_{P 2}^{j} t_{j}}$ and $n$ a finite integer $\left(U U^{\dagger}=\mathbb{1}\right)$ in such a way that both $H_{P 1}$ and $H_{P 2}$ are physical Hamiltonians, then the simulation would be straightforward. (We call this type of mapping a physical unitary mapping.) For our two qubit case, one can always perform a physical unitary mapping with

$$
\begin{gathered}
U=e^{i \frac{\pi}{4} \sigma_{x}^{2}} e^{-i \frac{\pi}{4} \sigma_{y}^{1}} e^{-i \frac{\theta}{2} \sigma_{z}^{1} \sigma_{z}^{2}} e^{i \frac{\pi}{4} \sigma_{y}^{1}} e^{i \frac{\pi}{4} \sigma_{x}^{1}} e^{-i \frac{\pi}{4} \sigma_{x}^{2}} e^{-i \frac{\pi}{4} \sigma_{y}^{2}} e^{i \frac{\theta}{2} \sigma_{z}^{1} \sigma_{z}^{2}} e^{-i \frac{\pi}{4} \sigma_{x}^{1}} e^{i \frac{\pi}{4} \sigma_{y}^{2}}, \\
H_{P 1}=\frac{1}{2}\left(E-\sqrt{\Delta^{2}+V^{2}}\right) \sigma_{z}^{1}+\frac{1}{2}\left(E+\sqrt{\Delta^{2}+V^{2}}\right) \sigma_{z}^{2}
\end{gathered}
$$

with $E=2\left(\epsilon+\mathcal{E}_{k_{0}}\right), \Delta=2\left(\epsilon-\mathcal{E}_{k_{0}}\right)$, and $\cos \theta=1 / \sqrt{1+\delta^{2}}$ with $\delta=\left(\Delta+\sqrt{\Delta^{2}+V^{2}}\right) / V$.

In general, such a constrained transformation is not easily realized and one performs a Trotter decomposition

$$
e^{i \bar{H} t}=\left[e^{i \bar{H} s}\right]^{M}=\left[e^{i \bar{H}_{z} s} e^{i \bar{H}_{x y} s}+\mathcal{O}\left(s^{2}\right)\right]^{M}
$$

where $\bar{H}=\bar{H}_{z}+\bar{H}_{x y}$ with $\bar{H}_{x y}=\frac{V}{2}\left(\sigma_{x}^{1} \sigma_{x}^{2}+\sigma_{y}^{1} \sigma_{y}^{2}\right)$ and time slice $s=\frac{t}{M}$. On the other hand, one can easily perform a physical unitary mapping for $e^{i \bar{H}_{x y} s}$

$$
e^{i \bar{H}_{x y} s}=\bar{U} e^{i H_{P 1} s} \bar{U}^{\dagger}
$$

where $H_{P 1}=\frac{V}{2}\left(\sigma_{x}^{1}-\sigma_{y}^{2}\right)$ and

$$
\bar{U}=e^{i \frac{\pi}{4} \sigma_{x}^{2}} e^{-i \frac{\pi}{4} \sigma_{y}^{1}} e^{-i \frac{\pi}{4} \sigma_{z}^{1} \sigma_{z}^{2}} .
$$

Finally, the "string" one has to simulate with the quantum computer is

$$
\begin{aligned}
& \mathcal{A}(t) \simeq[S(s)]^{M} \sigma_{-}^{1}\left[S^{\dagger}(s)\right]^{M} \sigma_{+}^{1} \\
& S(s)=e^{i \bar{H}_{z} s} \bar{U} e^{i H_{P 1} s} \bar{U}^{\dagger} .
\end{aligned}
$$

and $G(t)=\langle\mathcal{A}(t)\rangle$. 


\section{CONCLUDING REMARKS}

We investigated the implementation of algorithms for the simulation of fermionic quantum systems, and gave an explicit mapping that relates the usual qubit of a quantum computer to the fermionic modes that we want to simulate. Our attention focused on the so-called sign problem. It is a problem appearing in attempts to simulate classically the dynamics of quantum systems. We reviewed the origin of this problem and showed how this problem is avoided in quantum computing simulation. The evolution of quantum computers are intrinsically quantum mechanical and this is the main difference with a classical computer that allows one to solve the sign problem. We studied sources of errors in a quantum computer, such as gate imperfections and the expansion of the evolution operator, and argued that they would not open a back door to a problem similar to the sign problem.

We gave a very general definition of what a model of quantum computation is. In particular and because of our particular interest, i.e., the simulation of fermion systems, we described the standard and the fermionic models ("Grassmann Chip"). These are, of course, not the only ones. Isomorphisms of $*$-algebras allow one to introduce more "esoteric"

models [9]. Indeed, there is nothing special about the spinless fermionic model of quantum computation. One could have used a "hard-core boson" model which admits, in principle, a realization in terms of $\mathrm{He}^{4}$ atoms. The key point is the implementation of the physical gates.

Our effort focused on the simulation of the dynamics of fermionic quantum systems. However other problems can be of interest: the thermodynamic or ground state properties of a Hamiltonian. Even if one had a quantum computer, it is not clear how to use it to efficiently compute these quantities. On the other hand, at present, no proof exists showing that this is not possible.

An approach that in principle could be used to compute the spectrum of a Hamiltonian $H$ (e.g., the ground state) or expectation values of arbitrary observables is the adiabatic "switching on" in conjunction with the Gell-Mann-Low theorem [45] of quantum field theory. 
The idea simply consists of introducing a fictitious Hamiltonian

$$
H_{\epsilon}(t)=H_{0}+f_{\epsilon}(t) H_{1}
$$

where both $H_{0}$ and $H_{1}$ are time independent operators $\left(H=H_{0}+H_{1}\right)$ and the scalar function $f_{\epsilon}(t)$ is such that $\lim _{t \rightarrow \pm \infty} f_{\epsilon}(t)=0$ and $\lim _{t \rightarrow 0} f_{\epsilon}(t)=1$, for an arbitrary adiabatic parameter $\epsilon$. In other words, $H_{\epsilon}(t=0)=H$ and $H_{\epsilon}(t= \pm \infty)=H_{0}$. $H_{0}$ is typically an operator whose spectrum is known, e.g., an arbitrary bilinear operator representing a mean-field solution of $H$ and whose eigenstates can be straightforwardly prepared (let's call it $\left.\left|\Phi_{0}\right\rangle\right)$. The Gell-Mann-Low theorem asserts that

$$
\lim _{\epsilon \rightarrow 0} \frac{U_{\epsilon}(0,-\infty)\left|\Phi_{0}\right\rangle}{\left\langle\Phi_{0}\left|U_{\epsilon}(0,-\infty)\right| \Phi_{0}\right\rangle}=\frac{\left|\Psi_{0}\right\rangle}{\left\langle\Phi_{0} \mid \Psi_{0}\right\rangle}
$$

if the state whose limit one is performing admits a series expansion in a coupling parameter

characterizing the strength of $H_{1}$. This formal device generates the eigenstate adiabatically connected to $\left|\Phi_{0}\right\rangle$. The theorem does not guarantee that if $\left|\Phi_{0}\right\rangle$ is the ground state of $H_{0}$ then $\left|\Psi_{0}\right\rangle$ is the ground state of $H$. If the conditions of the theorem are satisfied then computation of the spectrum of $H$ is straightforward. To our knowledge this approach has never been implemented in practice.

The work presented here is only a first step in a program investigating the simulation of quantum systems using quantum computers. We have given a rather explicit algorithm for a simple problem and we will increase the complexity of the problems in the work to come. An interesting problem would be to provide algorithms to test for superconductivity in systems such as the Hubbard model. Such simulations using classical computers cannot unequivocally answer this important question because of the sign problem, but a quantum computer could.

\section{ACKNOWLEDGMENTS}

We acknowledge the Aspen Center for Physics for its kind hospitality while part of this work was performed. Work at Los Alamos is sponsored by the US DOE under contract 
W-7405-ENG-36. 


\section{APPENDIX: TOY PROBLEM: EXACT SOLUTION}

We can rewrite our original Hamiltonian, Eq. 25, in the wave number representation:

1. Kinetic energy:

$$
T=-\mathcal{T} \sum_{i=1}^{n}\left(c_{i}^{\dagger} c_{i+1}+c_{i+1}^{\dagger} c_{i}\right)=-\frac{\mathcal{T}}{n} \sum_{k, k^{\prime}, i} e^{i\left(k^{\prime}-k\right) R_{i}}\left(e^{i k^{\prime} a}+e^{-i k a}\right) c_{k}^{\dagger} c_{k^{\prime}}
$$

Thus,

$$
T=\sum_{i=1}^{n} \mathcal{E}_{k_{i}} c_{k_{i}}^{\dagger} c_{k_{i}}
$$

where $\mathcal{E}_{k}=-2 \mathcal{T} \cos k a$

2. Hybridization energy:

$$
H_{h y b}=\frac{V}{\sqrt{n}} \sum_{i=1}^{n}\left(c_{i}^{\dagger} b+b^{\dagger} c_{i}\right)=\frac{V}{n} \sum_{k, i}\left(e^{-i k R_{i}} c_{k}^{\dagger} b+e^{i k R_{i}} b^{\dagger} c_{k}\right)
$$

Therefore,

$$
H_{h y b}=V \sum_{k}\left(c_{k}^{\dagger} b+b^{\dagger} c_{k}\right) \delta_{k 0}
$$

Thus, in the wave number representation, the total Hamiltonian reads

$$
H=\sum_{i=0}^{n-1} \mathcal{E}_{k_{i}} c_{k_{i}}^{\dagger} c_{k_{i}}+\epsilon b^{\dagger} b+V \sum_{i=0}^{n-1}\left(c_{k_{i}}^{\dagger} b+b^{\dagger} c_{k_{i}}\right) \delta_{k_{i} 0}
$$

The quantization of $k$ quantizes the single particle energy spectrum $\mathcal{E}_{k}$. For example, for an $n=4$ site ring, the allowed $k$ values and energies $\mathcal{E}_{k}$ are shown in Fig. $\mathbb{1}$. 


\section{FIGURES}

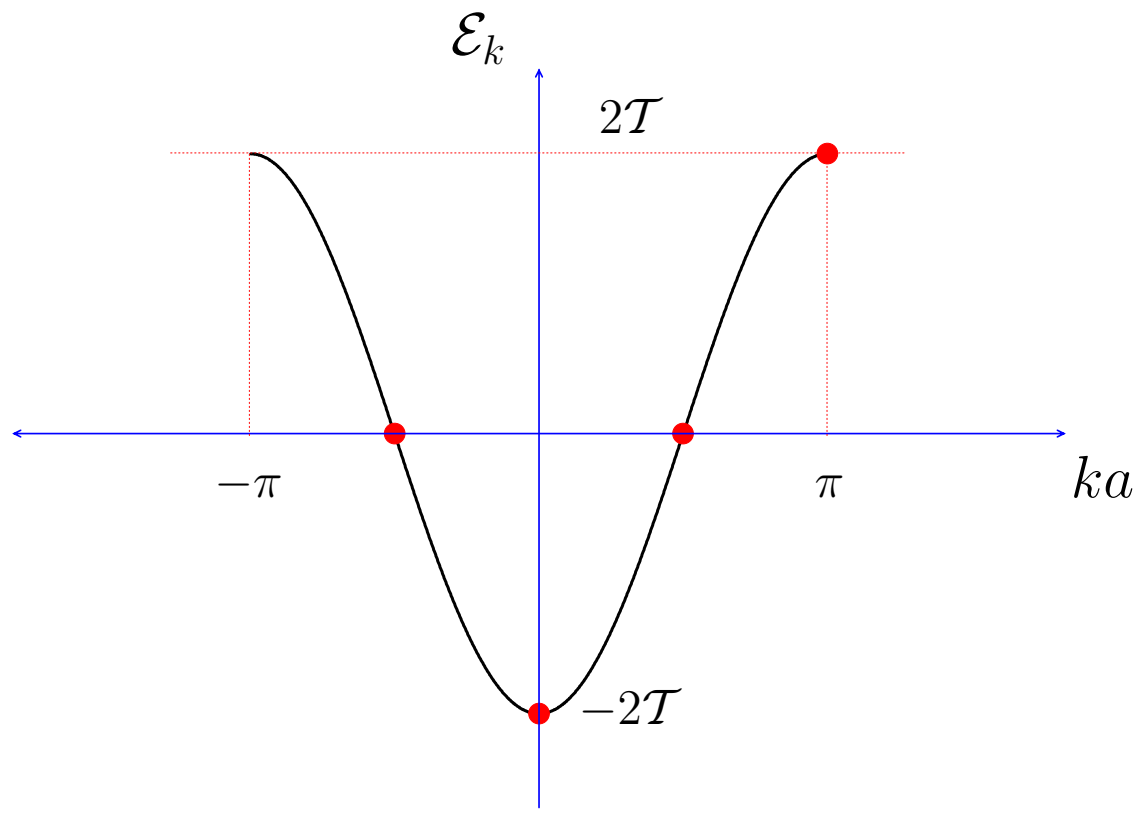

FIG. 1. Single-particle energy spectrum for an $n=4$ site ring.

Its ground state with $n_{e}=3$ is

$$
|F S\rangle=c_{k_{0}}^{\dagger} c_{k_{1}}^{\dagger} c_{k_{2}}^{\dagger}|0\rangle \text {, with } k_{0}=0, k_{1}=\frac{\pi}{2} \text {, and } k_{2}=-\frac{\pi}{2} \text {. }
$$

The Heisenberg equations of motion are:

$$
\begin{aligned}
& \dot{b}^{\dagger}=i\left[H, b^{\dagger}\right] \\
& \dot{c}_{k}^{\dagger}=i\left[H, c_{k}^{\dagger}\right],
\end{aligned}
$$

where $[A, B]=A B-B A$. These commutators equal

$$
\begin{aligned}
& {\left[H, b^{\dagger}\right]=\epsilon b^{\dagger}+V c_{k}^{\dagger} \delta_{k 0}} \\
& {\left[H, c_{k}^{\dagger}\right]=\mathcal{E}_{k} c_{k}^{\dagger}+V b^{\dagger} \delta_{k 0} .}
\end{aligned}
$$

Clearly, one can distinguish two cases:

1) $k \neq 0$ :

$$
c_{k}^{\dagger}(t)=e^{i \mathcal{E}_{k} t} c_{k}^{\dagger}(0)=e^{-i 2 t \mathcal{T} \cos k a} c_{k}^{\dagger}(0)
$$


2) $k=0\left(\mathcal{E}_{0}=-2 \mathcal{T}\right)$ :

$$
\begin{aligned}
& -i \dot{b}^{\dagger}=\epsilon b^{\dagger}+V c_{0}^{\dagger} \\
& -i \dot{c}_{0}^{\dagger}=\mathcal{E}_{0} c_{0}^{\dagger}+V b^{\dagger},
\end{aligned}
$$

or in matrix notation

$$
\left(\begin{array}{c}
\dot{b}^{\dagger} \\
\dot{c}_{0}^{\dagger}
\end{array}\right)=i\left(\begin{array}{cc}
\epsilon & V \\
V & \mathcal{E}_{0}
\end{array}\right) \cdot\left(\begin{array}{c}
b^{\dagger} \\
c_{0}^{\dagger}
\end{array}\right)
$$

The solution of these first-order differential equations are

$$
\left(\begin{array}{c}
b^{\dagger}(t) \\
c_{0}^{\dagger}(t)
\end{array}\right)=\exp \left[i t\left(\begin{array}{cc}
\epsilon & V \\
V & \mathcal{E}_{0}
\end{array}\right)\right] \cdot\left(\begin{array}{c}
b^{\dagger}(0) \\
c_{0}^{\dagger}(0)
\end{array}\right)
$$

From elementary matrix algebra

$$
\mathcal{U}(t)=\exp \left[i t\left(\begin{array}{cc}
\epsilon & V \\
V & \mathcal{E}_{0}
\end{array}\right)\right]=\left(\begin{array}{cc}
x & -y \\
y & x
\end{array}\right)\left(\begin{array}{cc}
e^{i t \lambda_{1}} & 0 \\
0 & e^{i t \lambda_{2}}
\end{array}\right)\left(\begin{array}{cc}
x & y \\
-y & x
\end{array}\right)
$$

where $\lambda_{1,2}$ are the eigenvalues of $\left(\begin{array}{cc}\epsilon & V \\ V & \mathcal{E}_{0}\end{array}\right)$ with corresponding eigenvectors $\left(\begin{array}{l}x \\ y\end{array}\right)$ and $\left(\begin{array}{c}-y \\ x\end{array}\right)$, and $x^{2}+y^{2}=1$. After elementary algebraic manipulations:

$$
\lambda_{1,2}=\frac{\epsilon+\mathcal{E}_{0}}{2} \pm \sqrt{\left(\frac{\epsilon-\mathcal{E}_{0}}{2}\right)^{2}+V^{2}}
$$

and

$$
\begin{aligned}
& x=\frac{V}{\sqrt{\left(\lambda_{1}-\epsilon\right)^{2}+V^{2}}} \\
& y=\frac{V}{\sqrt{\left(\lambda_{2}-\epsilon\right)^{2}+V^{2}}} .
\end{aligned}
$$

Thus, the operator $b(t)$ is expressible as

$$
b(t)=\mathcal{U}_{11}^{*}(t) b(0)+\mathcal{U}_{12}^{*}(t) c_{0}(0)
$$

where 


$$
\begin{aligned}
& \mathcal{U}_{11}^{*}(t)=x^{2} e^{-i t \lambda_{1}}+y^{2} e^{-i t \lambda_{2}} \\
& \mathcal{U}_{12}^{*}(t)=x y\left(e^{-i t \lambda_{1}}-e^{-i t \lambda_{2}}\right),
\end{aligned}
$$

and $\operatorname{det} \mathcal{U}(t)=e^{i t\left(\lambda_{1}+\lambda_{2}\right)}=e^{i t\left(\epsilon+\mathcal{E}_{0}\right)}$.

Finally, $G(t)$ is simply given by

$$
G(t)=\mathcal{U}_{11}^{*}(t)
$$

$|G(t)|^{2}=x^{4}+y^{4}+2 x^{2} y^{2} \cos \left(\lambda_{1}-\lambda_{2}\right) t$ and is plotted in Fig. 2.

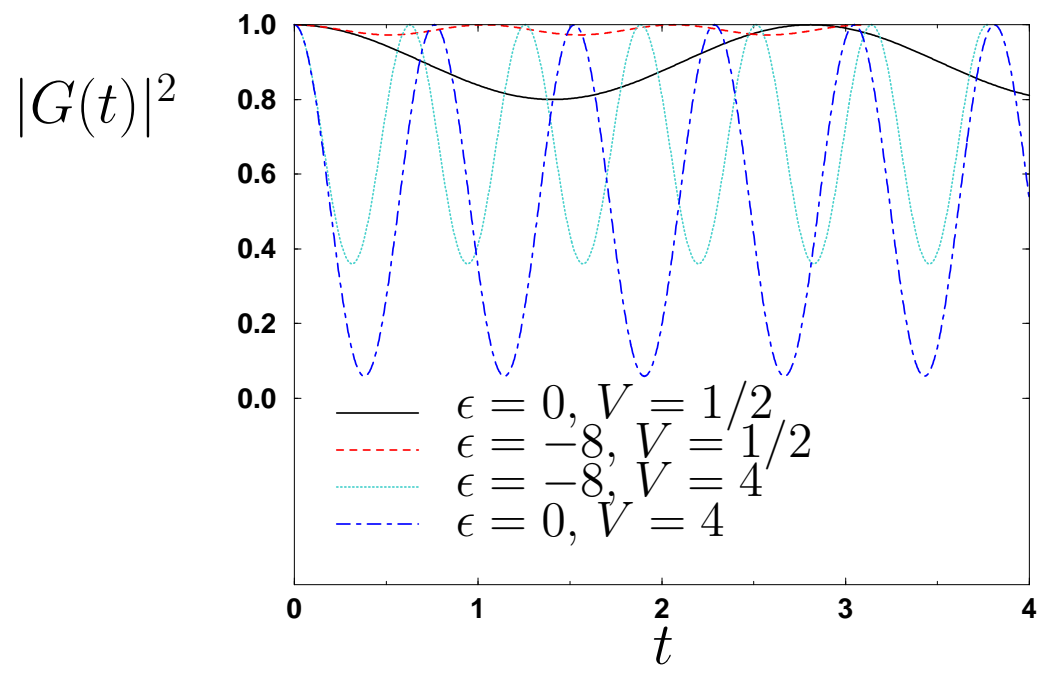

FIG. 2. $|G(t)|^{2}$ for different values of the parameters $\left(\mathcal{E}_{0}=-2\right)$.

Note that $G(t)$ is independent of the number of fermions present in the initial state $|\Psi(0)\rangle$. This is why this toy problem reduces to a two qubit problem. 


\section{REFERENCES}

[1] P. W. Shor, SIAM J. Comput. 26, 1484 (1997).

[2] L. K. Grover, Phys. Rev. Lett. 79, 325 (1997).

[3] R. P. Feynman, Int. J. Theor. Phys. 21, 467 (1982).

[4] D. S. Abrams and S. Lloyd, Phys. Rev. Lett. 79, 2586 (1997).

[5] M. H. Kalos, D. Levesque, and J. Verlet, Phys. Rev. A 9, 2178 (1974).

[6] D. M. Ceperley, Rev. Mod. Phys. 67, 279 (1995).

[7] D. M. Ceperley, in Recent Progress in Many-Body Theories, edited by E. Schachinger, H. Mitter, and M. Sormann (Plenum Press, New York, 1995), Vol. 4, pp. 455-470.

[8] In two spatial dimensions, other possibilities for quantum statistics emerge. For example, fractional statistics for particles called anyons interpolates continuously between bosons and fermions.

[9] C. D. Batista and G. Ortiz, cond-mat/0008374 (unpublished).

[10] From the point of view of complexity theory, an algorithm is efficient if it scales polynomially in time.

[11] G. Ortiz, D. M. Ceperley, and R. M. Martin, Phys. Rev. Lett. 71, 2777 (1993).

[12] J. Bonča and J. E. Gubernatis, Phys. Rev. E 53, 6504 (1996).

[13] M. Jarrell and J. E. Gubernatis, Phys. Rep. 269, 133 (1996).

[14] D. Deutsch, Proc. R. Soc. Lond. A 400, 97 (1985).

[15] We thank E. Fradkin for providing us with a preprint that circulated on April's fool day in 1983, where the term Grassmann chip is used as a joke. The manuscript is "Monte Carlo Simulation of a Realistic Unified Gauge Theory," by A. Chodos and J. Rabin (unpublished). 
[16] L. Onsager, Phys. Rev. 65, 117 (1944).

[17] S. Bravyi and A. Kitaev, quant-ph/0003137 (unpublished).

[18] R. P. Feynman and A. R. Hibbs, Quantum Mechanics and Path Integrals (McGraw-Hill, New York, 1965).

[19] The system is composed of $N_{e}$ particles moving in $d$ spatial dimensions $(\hbar=m=e=1)$, and a generic point in a flat Cartesian manifold of dimension $D=d N_{e}$ is represented by $\mathcal{R}=\left(\mathbf{r}_{1}, \cdots, \mathbf{r}_{N_{e}}\right) . V(\mathcal{R})$ is the potential energy operator and $p_{i}$ is particle's $i$ canonical momentum.

[20] J. W. Negele and H. Orland, Quantum Many-Particle Systems (Addison-Wesley, Redwood City, 1988).

[21] W. von der Linden, Phys. Rep. 220, 53 (1992).

[22] S. Lloyd, Science 273, 1073 (1996).

[23] E. Knill and R. Laflamme, quant-ph/9909094 (unpublished).

[24] A. Barenco et al., Phys. Rev. A 52, 3457 (1995).

[25] D. DiVincenzo, Phys. Rev. A 51, 1015 (1995).

[26] From the theory of computation point of view it is necessary to make additional assumptions on how the functions may be prescribed. In particular the functions themselves must be classically computable in a suitable sense. This problem is avoided by permitting only a finite set of quantum gates instead of continuously controllable Hamiltonians.

[27] A. Peres, Quantum Theory: Concepts and Methods (Kluwer Academic Publishers, Dordrecht, The Netherlands, 1998).

[28] E. Bernstein and U. Vazirani, SIAM J. Comput. 26, 1411 (1997).

[29] R. Cleve, quant-ph/9906111 (unpublished). 
[30] D. Aharonov, quant-ph/9812037 (unpublished).

[31] P. Jordan and E. Wigner, Z. Phys. 47, 631 (1928).

[32] E. Fradkin, Phys. Rev. Lett. 63, 322 (1989).

[33] L. Huerta and J. Zanelli, Phys. Rev. Lett. 71, 3622 (1993).

[34] M. Guerrero, G. Ortiz, and J. E. Gubernatis, Phys. Rev. B 59, 1706 (1999).

[35] A. Y. Kitaev, quant-ph/9511026 (unpublished).

[36] B. M. Terhal and D. P. DiVincenzo, Phys. Rev. A 61, 2301 (2000).

[37] E. Knill and R. Laflamme, Phys. Rev. Lett. 81, 5672 (1998).

[38] P. W. Shor, Phys. Rev. A 52, 2493 (1995).

[39] A. Steane, Proc. R. Soc. Lond. A 452, 2551 (1996).

[40] P. W. Shor, in Proceedings of the Symposium on the Foundations of Computer Science, edited by ? (IEEE press, Los Alamitos, California, 1996), pp. 56-65.

[41] D. Aharonov and M. Ben-Or, in Proceedings of the 29th Annual ACM Symposium on the Theory of Computation (STOC) (ACM Press, New York, New York, 1996), pp. $176-188$.

[42] A. Y. Kitaev, in Quantum Communication, Computing and Measurement, edited by O. Hirota, A. S. Holevo, and C. M. Caves (Plenum Press, New York, 1997).

[43] E. Knill, R. Laflamme, and W. H. Zurek, Science 279, 342 (1998).

[44] J. Preskill, Proc. R. Soc. Lond. A 454, 385 (1998).

[45] M. Gell-Mann and F. Low, Phys. Rev. 84, 350 (1951). 